\title{
Hepatitis A Outbreak with the Concurrence of Salmonella Typhi and Salmonella Poona Infection in Children of Urban Vellore, South India
}

\author{
Manikandan Srinivasan, ${ }^{1} \dagger$ Kulandaipalayam Natarajan Sindhu, ${ }^{1} \dagger$ Senthil J. Kumar, ${ }^{2}$ Priya Abraham, ${ }^{3}$ Shalini Anandan, ${ }^{4}$ \\ Veeraraghavan Balaji, ${ }^{4}$ Venkata Raghava Mohan, ${ }^{2}$ Gagandeep Kang, ${ }^{1}$ and Jacob John ${ }^{2 \star}$ \\ ${ }^{1}$ Division of Gastrointestinal Sciences, Wellcome Trust Research Laboratory, Christian Medical College, Vellore, India; ${ }^{2}$ Department of Community \\ Health, Christian Medical College, Vellore, India; ${ }^{3}$ Department of Clinical Virology, Christian Medical College, Vellore, India; ${ }^{4}$ Department of Clinical \\ Microbiology, Christian Medical College, Vellore, India
}

\begin{abstract}
We investigated an outbreak of hepatitis A infection among children in an urban settlement of Vellore, South India. A total of 58 cases of jaundice were reported between April and August 2019. Sera from children who presented with jaundice were tested for hepatitis A virus (HAV) IgM. HAV IgM was positive in $18(94.7 \%)$ of the 19 cases tested. These children also received a blood culture at the same time, as a part of the ongoing Surveillance for Enteric Fever in India (SEFI). Blood cultures from three children with confirmed hepatitis A infection grew Salmonella sp.: two with Salmonella Typhi and one with Salmonella Poona. Salmonella Poona is being reported for the first time from India. The overall hospitalization rate during the outbreak was $21 \%$. Outbreaks of hepatitis A continue to occur with substantial morbidity in children from endemic settings, with notable emergence of other concurrent enteric infections, thereby warranting continued surveillance.
\end{abstract}

Outbreaks of hepatitis A virus (HAV) infection continue to be reported from parts of India, transitioning from hyper- to intermediate endemicity. ${ }^{1-4}$ Populations residing in hyperendemic areas are exposed to HAV at an earlier age during childhood, presenting mostly as asymptomatic infections, thereby acquiring protection from natural infection. However, in communities that are transitioning from a hyper-endemic to an intermediate-endemic state for HAV, there has been a resultant decrease in HAV circulation. ${ }^{5}$ This has brought in a shift in the age at infection from early childhood to later ages, in those whom the disease tends to be severe. ${ }^{6}$ In this context, many parts of India which are in the process of improving their water and sewage facilities face a risk of periodic outbreaks of HAV. As and when the threshold in the build-up of susceptibles has been attained, along with concurrent disruptions in water and sewage systems, conditions become conducive, favoring the feco-oral transmission of enteric pathogens. Hepatitis A virus and Salmonella sp. share similar routes of transmission and may coinfect individuals at risk. We report here an outbreak of hepatitis A with concomitant salmonellosis from an urban settlement of Vellore in South India between April and August 2019, a locality which previously reported an outbreak of HAV in $2006{ }^{2}$

Surveillance for Enteric Fever in India (SEFI) was established in October 2017 to estimate the age-specific incidence of typhoid fever in Indian children aged between 6 months and 15 years in community-based cohorts across four different sites in India by active surveillance for fever. ${ }^{7}$ The cohort at Vellore comprises 6,000 children from four contiguous semi-urban settlements of Vellore town (Chinnallapuram [CAP], Ramnaickanpalayam [RNP], Kaspa, and Vasanthapuram [VSPM]). The Institutional Review Board (IRB) of Christian Medical College, Vellore, approved the community surveillance for febrile illness under SEFI (IRB Min No: 10,393, dated November 30, 2016).

\footnotetext{
*Address correspondence to Jacob John, Department of Community Health, Christian Medical College, Ida Scudder road, Vellore 632 004, India. E-mail: jacob@cmcsph.org

†These authors contributed equally to this work.
}

In June 2019, clustering of jaundice cases was observed in the Vellore cohort of SEFI, and therefore, an outbreak investigation was conducted, interviewing households, children and adults, from clusters of cases identified within the four areas of Vellore cohort. A case was defined as any child or family member with a history of jaundice between April and August 2019, with no prior history of liver disease. The SEFI field research assistants who performed weekly surveillance elicited history of jaundice from 6,212 individuals in 1,239 families. Of the 6,212 individuals, 2,480 (40\%) were children aged less than 15 years. Cases identified in the survey were further contacted by the study physician to confirm jaundice, and a detailed history was collected. Subjects who consented were tested for IgM anti-HAV and IgM antiHepatitis E virus (HEV) at the department of Clinical Virology, Christian Medical College, Vellore. An epidemic curve depicting the weekly occurrence of cases was constructed using Stata 15 (StataCorp. [2017]. Stata Statistical Software: Release 15. College Station, TX: StataCorp LLC) (Figure 1). A spot map of cases of hepatitis was plotted using Geographical Information System coordinates captured during the investigation (Figure 2).

Between April and August 2019, we identified 58 cases of jaundice using the surveillance case definition. The baseline characteristics of these 58 cases are shown in Table 1. Of the 58 cases, 57 were children aged less than 15 years, with one adult aged 19 years. The attack rate of jaundice in children aged less than 5 years, $5-10$ years, and $10-15$ years was $1.5 \%$ (8/537), $2.8 \%(28 / 1,005)$, and $2.4 \%$ (22/938), respectively. Clustering within eight streets of the study area was observed in 31 of the 58 cases, with eight cases from a single street of RNP. The clustering of cases in time is shown in Figure 1, with a primary peak at week 4 consisting of nine cases, followed by secondary peaks at week 8 with 6 cases. Following rains in the later part of July, the monsoon period for Vellore and the rest of south India, the epidemic continued resulting in two additional peaks at weeks 13 and 15, with seven cases each. Clustering of 14 cases was seen among individuals sharing the same compound, with one particular dwelling reporting four cases. Another 10 cases were clustered, with two cases each being reported from five houses. The time interval between the primary and secondary cases within these houses ranged 


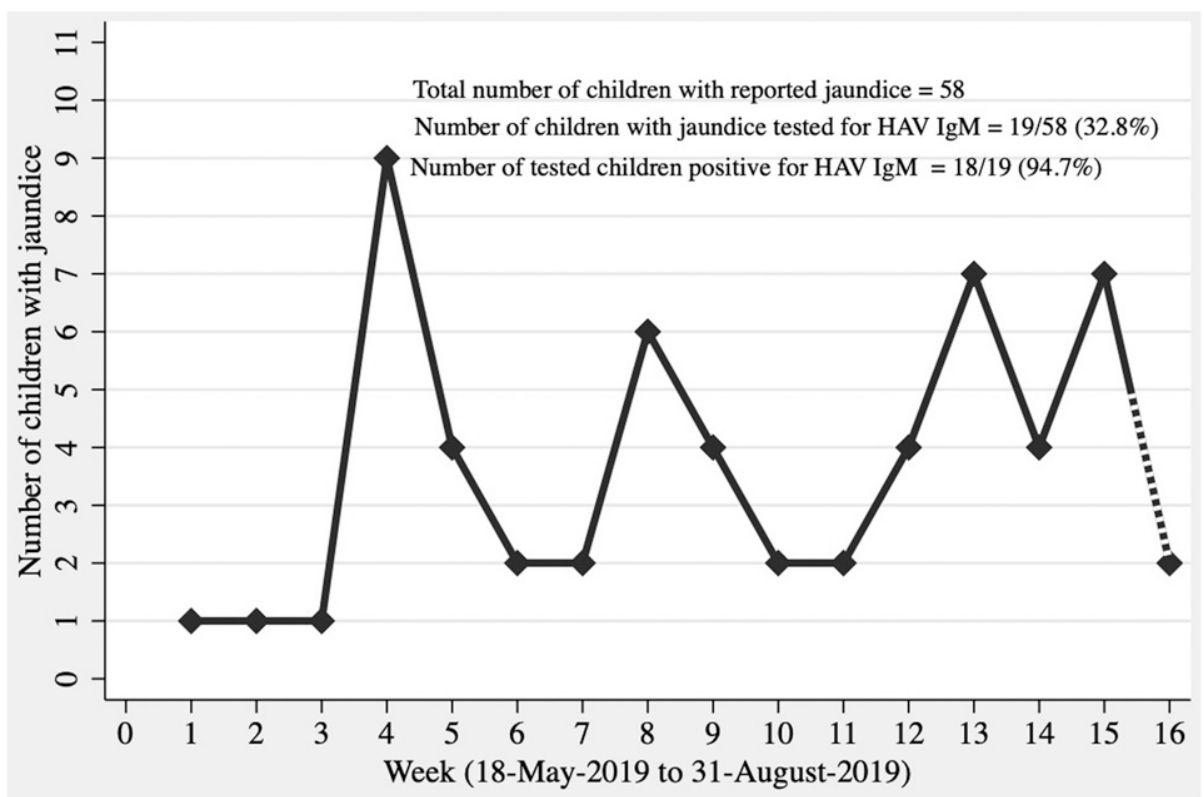

FIGURE 1. Epidemic curve showing the number of children with hepatitis in the Surveillance for Enteric Fever in India cohort between May 18,2019 and August 10, 2019.

between 4 and 40 days. Twelve (20.7\%) of 58 cases with jaundice were hospitalized. Liver function tests of children less than 6 years of age showed a 7 - to 33 -fold increase in alanine transaminase (ALT) from baseline $(35 \mathrm{U} / \mathrm{L})$, whereas older children (10-13 years) showed a 13- to 73-fold increase. Of those tested for IgM HAV (19/58) and IgM HEV (11/58), 18/19 $(94.7 \%)$ were positive for IgM HAV, and all 11 tested negative for IgM HEV. Between April and August 2019, 64 typhoid fevers were detected in the Vellore cohort of SEFI. As a part of SEFI protocol, blood cultures are performed for children with fever lasting for more than 3 days (defined as suspected typhoid fever), and thereby, 24 of 58 cases of jaundice received a blood culture during the same episode of illness when they presented with jaundice. Two children grew Salmonella Typhi and one Salmonella Poona, with all three being concurrently positive for HAV IgM. The ultrasonogram abdomen of one particular child, coinfected with HAV and S. Typhi, showed a thickened and edematous outer wall of the gall bladder.

Children younger than 5 years are at a high risk for HAV infection in endemic settings. Our findings show a low attack rate in the under-fives because children under-five are often asymptomatic following HAV infection and can be identified only by serosurveys. In older children, HAV infection tends to be clinically severe, leading to very high ALT levels. ${ }^{5}$ Drinking water sampling carried out between April and July 2019 from 306 randomly selected SEFI households revealed coliform colonies $>10,000$ per $100 \mathrm{~mL}$ in 229 (74.8\%) households, and none of the samples showed the presence of residual chlorine $(<0.5 \mathrm{ppm})$, indicating the dangerously poor standards in the quality of drinking water supplied through the public distribution system in the area. Around $86 \%$ of SEFI households depend on the public distribution system for drinking water, the supply often being intermittent. Furthermore, 15\% of these households lacked proper sanitation, with their toilets directly connecting to open drains. The sewage drains and drinking water pipes in this area run in parallel and close proximity (Figure 2). Negative suction in water

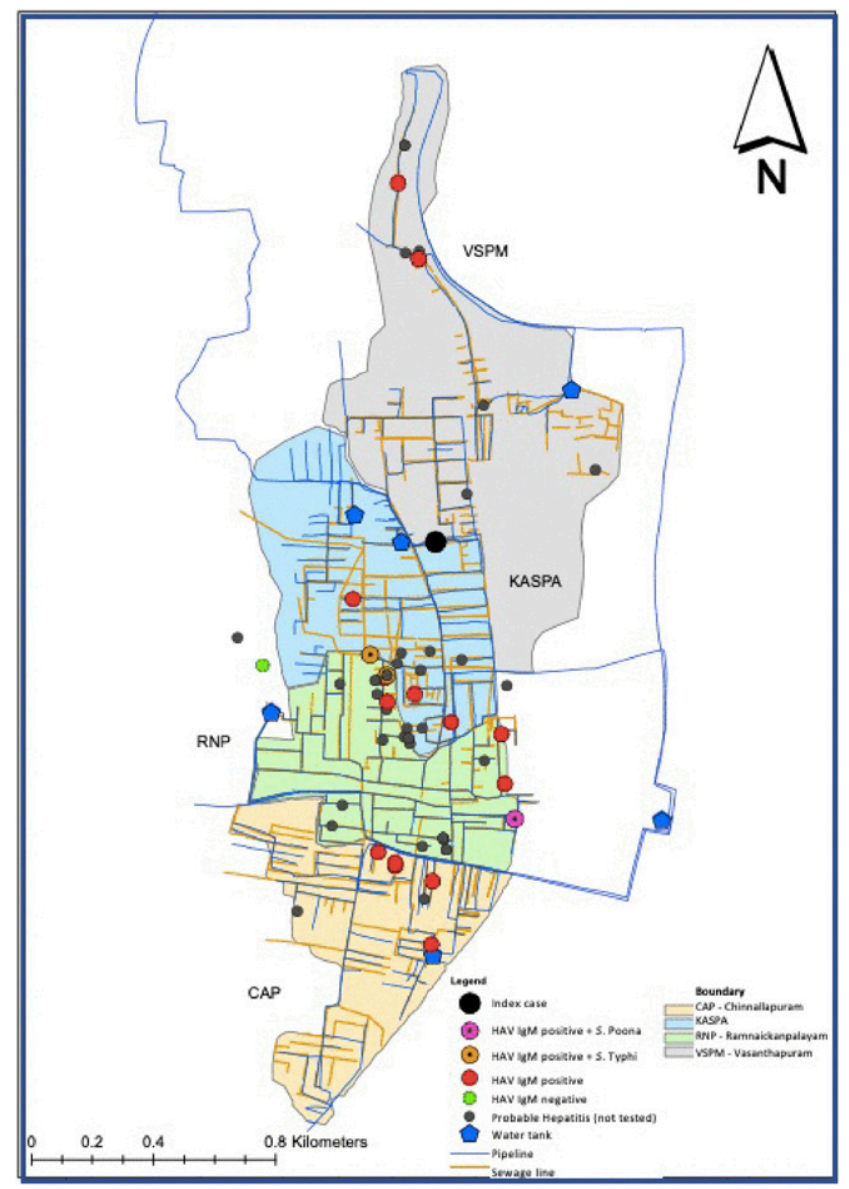

FIGURE 2. Spot map showing the cases of hepatitis along with those positive for hepatitis A virus IgM and Salmonella Typhi/Salmonella Poona in the Surveillance for Enteric Fever in India study site using a geographic information system. This figure appears in color at www.ajtmh.org. 
TABLE 1

Baseline characteristics of the children with hepatitis in the Surveillance for Enteric Fever in India cohort $(N=58)$

\begin{tabular}{|c|c|c|}
\hline & & $N=58$ \\
\hline \multirow[t]{3}{*}{ Age (years) } & $<5$ & $8(13.8)$ \\
\hline & $5-10$ & $28(48.3)$ \\
\hline & $>10$ & $22(37.9)$ \\
\hline \multirow{2}{*}{ Gender } & Male & $26(44.8)$ \\
\hline & Female & $32(55.2)$ \\
\hline \multirow[t]{6}{*}{ Clinical features } & Jaundice & $58(100)$ \\
\hline & Fever & 46 (79.3) \\
\hline & Abdominal pain & 45 (77.6) \\
\hline & Loss of appetite & $41(70.7)$ \\
\hline & Vomiting & $43(74.1)$ \\
\hline & Loose stools & $14(24.1)$ \\
\hline \multirow[t]{2}{*}{ HAV IgM testing } & Number of children tested & 19 (32.8) \\
\hline & $\begin{array}{l}\text { Number of children positive among those } \\
\text { tested }\end{array}$ & $18 / 19(94.7)$ \\
\hline \multirow[t]{3}{*}{ Blood culture } & $\begin{array}{l}\text { Number of children with jaundice who had } \\
\text { a concurrent blood culture }\end{array}$ & $24(41.4)$ \\
\hline & $\begin{array}{l}\text { Number of children positive for } \\
\text { Salmonella Typhi }\end{array}$ & $2 / 24(8.3)^{\star}$ \\
\hline & $\begin{array}{l}\text { Number of children positive for } \\
\text { Salmonella Poona }\end{array}$ & $1 / 24(4.2) \dagger$ \\
\hline Hospitalization & Number of children hospitalized & $12(20.7)$ \\
\hline \multirow[t]{5}{*}{ Epidemiological characteristics } & Source of drinking water & \\
\hline & a. Public distribution system & $53(91.4)$ \\
\hline & b. Bottled water/packaged water & $5(8.6)$ \\
\hline & $\begin{array}{l}\text { Consumption of locally prepared ice } \\
\text { sticks before illness }\end{array}$ & $48(82.8)$ \\
\hline & History of contact with a case of jaundice & $14(24.1)$ \\
\hline
\end{tabular}

$\dagger$ This child was positive for HAV IgM and S. Poona.

pipelines due to intermittent water supply and the pre-existing breaks in the older pipelines can facilitate contamination of water with sewage lines. ${ }^{8}$ Considering the inadequately engineered water supply and acute phase shedding of HAV in stool, with the incubation period being 28 days for HAV, the incident cases in the primary wave possibly served as the source of infection for the secondary wave, and hence the peaks at weeks 8,13 , and $15 .^{5}$ Thus, the unplanned urbanization in the area with no sewage

TABLE 2

Clinical characteristics of the three children positive for HAV IgM and concurrent S.Typhi/S. Poona

\begin{tabular}{|c|c|c|c|}
\hline Characteristic & Child 1: hepatitis A with S. Typhi & Child 2: hepatitis A with S. Typhi & Child 3: hepatitis A with S. Poona \\
\hline Age (completed years) & 10 & 10 & 12 \\
\hline Gender & Male & Male & Male \\
\hline $\begin{array}{l}\text { First presenting symptom (duration in } \\
\text { days) }\end{array}$ & Fever (8) & Fever (7) & Fever (5) \\
\hline $\begin{array}{l}\text { Time of presentation of jaundice during } \\
\text { the illness episode }\end{array}$ & First week & First week & Second week \\
\hline Other symptoms & $\begin{array}{l}\text { Abdominal pain, loss of appetite, } \\
\text { and vomiting }\end{array}$ & $\begin{array}{l}\text { Abdominal pain, loss of } \\
\text { appetite, and } \\
\text { vomiting }\end{array}$ & Abdominal pain and loss of appetite \\
\hline Hospitalization & Yes & No & No \\
\hline HAV IgM & Positive & Positive & Positive \\
\hline Blood culture & S. Typhi & S. Typhi & S. Poona \\
\hline \multicolumn{4}{|l|}{ Liver function test } \\
\hline Total bilirubin (TB) (mg/dL) & 6.2 & NA & 5.8 \\
\hline Direct bilirubin (DB) (mg/dL) & 5.7 & & 3.7 \\
\hline AST (U/L) & 127 & NA & 371 \\
\hline ALT (U/L) & 578 & NA & 1,027 \\
\hline $\operatorname{ALP}(\mathrm{U} / \mathrm{L})$ & 282 & NA & 307 \\
\hline Ultrasonogram of abdomen & $\begin{array}{l}\text { Distended gallbladder } \\
\text { with thickened and edematous } \\
\text { outer wall }\end{array}$ & NA & NA \\
\hline Treatment taken & $\begin{array}{l}\text { IV ampicillin, gentamycin, } \\
\text { metronidazole, cefotaxime for } 2 \\
\text { days, followed by oral cefixime for } 15 \\
\text { days }\end{array}$ & $\begin{array}{l}\text { Oral azithromycin for } 12 \\
\text { days }\end{array}$ & Oral azithromycin for 5 days \\
\hline Outcome & Recovered without sequela & $\begin{array}{l}\text { Recovered without } \\
\text { sequela }\end{array}$ & Recovered without sequela \\
\hline
\end{tabular}


treatment propagated the epidemic, lengthening it to last for 16 weeks and beyond. Furthermore, poor or no chlorination at the level of water entry into the public distribution system could have resulted in the thriving of various enteric pathogens, thus leading to the co-occurrence of HAV with Salmonella sp. as seen in the three children during the epidemic. There have been case reports on HAV and S. Typhi coinfection in children from other Indian settings, but not with $S$. Poona., ${ }^{9,10}$

The child coinfected with S. Typhi and HAV presented with inflammatory changes in the gall bladder, and it is unclear whether inflammation of the gallbladder in HAV infection favors the persistence of $S$. Typhi, predisposing them to the carrier state. ${ }^{11-13}$ This is the first report from India reporting a coinfection of HAV and $S$. Poona in a child. Outbreaks of $S$. Poona, an invasive non-typhoidal Salmonella, have been reported mainly from the Western world, with hospitalization and case fatality rates of $22.5 \%$ and $0.6 \%$, respectively, in the United States. ${ }^{14-16}$ A hospital-based study has shown that hepatitis A infections account for about $50 \%$ of acute hepatitis and acute liver failure cases in Indian children presenting to a tertiary care center. ${ }^{17}$ Although the population in endemic areas with poor sanitation acquire natural protection for hepatitis $A$ infection by early childhood, the occurrence of an outbreak with substantial pediatric hospitalization rate in this population raises concern.

Our findings highlight that hepatitis $A$ infection can present as sporadic outbreaks in communities with substandard water and sewage systems, concurrent with other enteric infections such as invasive salmonellosis. Thus, population-based surveillance for hepatitis $A$ is required in India, to identify populations and geographical regions at risk, and thereby potentially plan implementation strategies for hepatitis $A$ vaccination. While typhoid per se can present with hepatitis as a complication, although rarely, it is also plausible for the patient to present with hepatitis due to coinfection with HAV in settings similar to this. Continued surveillance is needed to carefully look for the emergence of salmonellosis occurring in concurrence with outbreaks of HAV and other enteric infections in urban settlements with poorly engineered water and sewage systems with dense population agglomerations.

Received October 7, 2019. Accepted for publication January 15, 2020. Published online March 30, 2020.

Acknowledgments: This outbreak investigation would have not been possible without the cooperation of the Surveillance for Enteric Fever in India (SEFI) study participants, the Chinnallapuram community, and the SEFI field research assistants. We would like to thank Prabhakar D. Moses, Anita Shirley David, Atrayee Nag, and Jacklin for their clinical support.

Financial support: This work was supported by the Wellcome Trust Research Laboratory, Division of Gastrointestinal Sciences, Christian Medical College, Vellore, India.

Authors' addresses: Manikandan Srinivasan, Kulandaipalayam Natarajan Sindhu, and Gagandeep Kang, Division of Gastrointestinal Sciences, Wellcome Trust Research Laboratory, Christian Medical College, Vellore, India, E-mails: manikandanmbbs06@gmail.com, sindhukn@cmcvellore.ac.in, and gkang@cmcvellore.ac.in. Senthil J. Kumar, Venkata Raghava Mohan, and Jacob John, Department of Community Health, Christian Medical College, Vellore, India, E-mails: senthilcmc2008@gmail.com, venkat@cmcvellore.ac.in, and jacob@ cmcsph.org. Priya Abraham, Department of Clinical Virology, Christian Medical College, Vellore, India, E-mail: priyaabraham@ cmcvellore.ac.in. Shalini Anandan and Veeraraghavan Balaji, Department of Clinical Microbiology, Christian Medical College, Vellore, India, E-mails: shalinianandan@cmcvellore.ac.in and vbalaji@ cmcvellore.ac.in.

\section{REFERENCES}

1. Rakesh P, Sherin D, Sankar H, Shaji M, Subhagan S, Salila S, 2014. Investigating a community-wide outbreak of hepatitis $A$ in India. J Glob Infect Dis 6: 59-64.

2. Sowmyanarayanan TV, Mukhopadhya A, Gladstone BP, Sarkar R, Kang G, 2008. Investigation of a hepatitis A outbreak in children in an urban slum in Vellore, Tamil Nadu, using geographic information systems. Indian J Med Res 128: 32-37.

3. Nandi B, Hadimani P, Arunachalam R, Ganjoo R, 2009. Spectrum of acute viral hepatitis in southern India. Med $J$ Armed Forces India 65: 7-9.

4. Gurav YK, Retheesh Babu G, Vinu KP, Lole KS, 2019. Suspected spread of hepatitis $A$ virus from a restaurant among adults in rural area of the Kerala state, India. Epidemiol Infect 147: e210.

5. Centre for Disease Control and Prevention, 2015. Immunology and Vaccine-Preventable Diseases-Pink Book-Hepatitis A. Available at: https://www.cdc.gov/vaccines/pubs/pinkbook/downloads/ hepa.pdf. Accessed August 6, 2019.

6. Franco E, Meleleo C, Serino L, Sorbara D, Zaratti L, 2012. Hepatitis $A$ : epidemiology and prevention in developing countries. World J Hepatol 4: 68-73.

7. John J, Bavdekar A, Rongsen-Chandola T, Dutta S, Kang G, 2018. Estimating the incidence of enteric fever in children in India: a multi-site, active fever surveillance of pediatric cohorts. BMC Public Health 18: 594.

8. Sarkar R, Prabhakar AT, Manickam S, Selvapandian D, Raghava MV, Kang G, Balraj V, 2007. Epidemiological investigation of an outbreak of acute diarrhoeal disease using geographic information systems. Trans R Soc Trop Med Hyg 101: 587-593.

9. Mishra D, Chaturvedi D, Mantan M, 2008. Typhoid fever and viral hepatitis. Indian J Pediatr 75: 509-510.

10. Zaki SA, Shanbag P, 2012. Co-existence of typhoid fever and hepatitis A. Ann Trop Med Public Health 5: 551.

11. Kaya S, Eskazan AE, Ay N, Baysal B, Bahadir MV, Onur A, Duymus $\mathrm{R}, 2013$. Acute acalculous cholecystitis due to viral hepatitis $A$. Case Rep Infect Dis 2013: 407182.

12. Ozaras R, Mert A, Yilmaz MH, Celik AD, Tabak F, Bilir M, Ozturk R, 2003. Acute viral cholecystitis due to hepatitis A virus infection. J Clin Gastroenterol 37: 79-81.

13. Gonzalez-Escobedo G, Marshall JM, Gunn JS, 2011. Chronic and acute infection of the gall bladder by Salmonella Typhi: understanding the carrier state. Nat Rev Microbiol 9: 9-14.

14. Hughes MH, Speller PJ, 1969. Salmonella poona in Hampshire. Lancet 293: 936-937.

15. Centre for Disease Control and Prevention, 2019. Multistate Outbreak of Salmonella Poona Infections Linked to Imported Cucumbers. Available at: https://www.cdc.gov/salmonella/Poona09-15/index.html. Accessed August 19, 2019.

16. Stone A, Shaffer M, Sautter RL, 1993. Salmonella poona infection and surveillance in a neonatal nursery. Am J Infect Control 21: 270-273.

17. Sood V, Lal BB, Gupta E, Khanna R, Siloliya MK, Alam S, 2019. Hepatitis A virus-related pediatric liver disease burden and its significance in the Indian subcontinent. Indian Pediatr 56: 741-744. 